\title{
STABILITY OF NONLINEAR FUNCTIONAL STOCHASTIC EVOLUTION EQUATIONS OF SECOND ORDER IN TIME
}

\author{
Tomás Caraballo, María J. Garrido-Atienza and José Real \\ Departamento de Ecuaciones Diferenciales y Análisis Numérico, \\ Universidad de Sevilla, Apdo. de Correos 1160, \\ 41080-Sevilla, Spain.
}

\begin{abstract}
Sufficient conditions for exponential mean square stability of solutions to delayed stochastic partial differential equations of second order in time are established. As a consequence of these results, the pathwise exponential stability of the system is also deduced. The stability results derived can be applied also to partial differential equations without hereditary characteristics. The results are illustrated with various examples.
\end{abstract}

\section{Introduction}

Stochastic differential delay equations and their asymptotic behaviour have been receiving much attention over the last years (see [1], [2], [5], [9], [7], [8], [10], [12], [13], and the references therein) since these retarded problems often appear in Physics, Biology, Engineering, etc...

The delays can enter in the formulations in very different ways, e.g., as a constant or variable delay, as a distributed one, or even some of them can appear in the model at the same time. However, it is possible to consider all of them under a unified formulation by using an appropriate differential functional equations. In this sense, we will carry out our analysis in a functional framework which will cover a wide variety of situations containing finite delays. 
There exists a wide literature concerning pathwise exponential stability of parabolic stochastic evolution equations (with and without delays). We mention here, amongst many others, Caraballo and Liu [4], Liu and Mao [11], Taniguchi [12], Taniguchi et al. [13] and the references therein. In the case without hereditary characteristics the problem of the asymptotic stability of delay stochastic partial differential equations of second order in time has been considered by Curtain [6], where one can find sufficient conditions for the exponential stability of the expected energy of the system, as well as for the exponential decay of the sample paths, when the main operator generates a strongly continuous contraction semigroup. In this paper, we shall develop the theory in a variational framework for non-linear operators in general.

We are going to consider the following model

$$
\left\{\begin{array}{l}
u^{\prime}(t)+\int_{0}^{t} A(s) u(s) \mathrm{d} s+\int_{0}^{t} B\left(s, u^{\prime}(s)\right) \mathrm{d} s=v_{0}+\int_{0}^{t} F\left(s, u_{s}, u_{s}^{\prime}\right) \mathrm{d} s \\
+\int_{0}^{t} G\left(s, u_{s}, u_{s}^{\prime}\right) \mathrm{d} W(s), t \geq 0, \\
u(0)=u_{0}, \\
u(t)=\varphi_{1}(t), u^{\prime}(t)=\varphi_{2}(t), \text { a.e. } t \in(-h, 0),
\end{array}\right.
$$

where $A$ is a family of linear operators and $B, F, G$ are appropriate nonlinear operators on some Hilbert spaces (usually both $F$ and $G$ will be first order partial derivative operators), $\varphi_{1}, \varphi_{2}$ are suitable delay functions and $h>0$ is a fixed constant.

In our recent work [3], the exponential behaviour of solutions of problem (1) has been analysed focusing on the case in which the operators are of zero order and when a particular family of nonlinear operators $B(t, \cdot)$ appears. Our aim in this work is to obtain some sufficient conditions ensuring exponential stability of the solution of problem (1) when $F$ and/or $G$ can eventually depend on the spatial derivatives of $u$, and $B(t, \cdot)$ is a general family of nonlinear operators.

Although the structure of this work paralells that of [3], needless to say that several differences will appear due to the different nature of operators appearing in the problem. Of course, our results are a consistent extension of those in [3] in the sense that, when the operators considered are of zero order, we recover the results in [3].

The content of the paper is as follows. In Section 2 we prove a result on the exponential stability in mean square for the solution of problem (1) when $F\left(t, u_{t}, u_{t}^{\prime}\right)$ and $G\left(t, u_{t}, u_{t}^{\prime}\right)$ do not depend on the spatial derivatives of $u$, being $B$ a general family of nonlinear operators. We also indicate how this result can be applied in some particular cases. As a consequence of the mean square stability, we obtain pathwise exponential stability. In Section 3 we establish a sufficient condition ensuring mean square stability for delay stochastic partial differential 
equations of second order in time in a more general situation: $F\left(t, u_{t}, u_{t}^{\prime}\right)$ and/or $G\left(t, u_{t}, u_{t}^{\prime}\right)$ may depend on the spatial derivatives of $u$. Moreover, in this section we will exhibit how we can prove a result on the pathwise exponential stability in this more general situation. In Section 4 we illustrate our theory with some examples, and, finally, some conclusions are included in the last section.

\section{Delays independent of the spatial derivatives}

Assume $\{\Omega, \mathcal{F}, P\}$ is a complete probability space with a normal filtration $\left\{\mathcal{F}_{t}\right\}_{t \geq 0}$, i.e., $\mathcal{F}_{0}$ contains the null sets in $\mathcal{F}$, and $\mathcal{F}_{t}=\cap_{s>t} \mathcal{F}_{s}$, for all $t \geq 0$. Denote $\mathcal{F}_{t}=\mathcal{F}_{0}$ for all $t \leq 0$.

Let us consider a real valued $\left\{\mathcal{F}_{t}\right\}$-Wiener process $\{W(t)\}_{t \geq 0}$.

Given real numbers $a<b$ and a separable Hilbert space $\mathcal{H}$, we will denote by $I^{2}(a, b ; \mathcal{H})$ the closed subspace of $L^{2}(\Omega \times(a, b), \mathcal{F} \otimes \mathcal{B}([a, b]), \mathrm{d} P \otimes \mathrm{d} t ; \mathcal{H})$ of all stochastic processes which are $\mathcal{F}_{t}$-adapted for almost every $t$ in $(a, b)$ (in what follows, a.e. $t$ ), where $\mathcal{B}([a, b])$ denotes the Borel $\sigma$-algebra of subsets in $[a, b]$. If $\varphi \in I^{2}(a, b ; \mathcal{H})$ we will write $|\varphi|_{I_{\mathcal{H}}^{2}}$ to denote the norm $|\varphi|_{I^{2}(a, b ; \mathcal{H})}$.

We denote by $L^{2}(\Omega ; C(a, b ; \mathcal{H}))$ the space of processes $X \in L^{2}(\Omega, \mathcal{F}, d P ; C(a, b ; \mathcal{H}))$ such that $X(t)$ is $\mathcal{F}_{t}$-measurable for each $t$ in $[a, b]$, where $C(a, b ; \mathcal{H})$ denotes the space of all continuous functions from $[a, b]$ into $\mathcal{H}$ equipped with supremum norm.

Let us fix $h>0$ and consider $T>0$. If we have a function $x \in C(-h, T ; \mathcal{H})$, for each $t \in[0, T]$ we denote by $x_{t} \in C(-h, 0 ; \mathcal{H})$ the function defined by $x_{t}(s)=x(t+s)$, $-h \leq s \leq 0$. Moreover, if $y \in L^{2}(-h, T ; \mathcal{H})$ we also denote by $y_{t} \in L^{2}(-h, 0 ; \mathcal{H})$, a.e. $t \in(0, T)$, the function defined by $y_{t}(s)=y(t+s)$, a.e. $s \in(-h, 0)$.

We will study the qualitative behaviour of the following delay stochastic functional equation:

$$
\left\{\begin{array}{l}
u \in I^{2}(-h, T ; V) \cap L^{2}(\Omega ; C(0, T ; V)), \text { for all } T>0, \\
v \in I^{2}(-h, T ; H) \cap L^{2}(\Omega ; C(0, T ; H)), \text { for all } T>0, \\
u^{\prime}(t)=v(t), t \in[0, T] \\
v(t)+\int_{0}^{t} A(s) u(s) \mathrm{d} s+\int_{0}^{t} B(s, v(s)) \mathrm{d} s=v_{0}+\int_{0}^{t} F_{0}\left(s, u_{s}, v_{s}\right) \mathrm{d} s \\
+\int_{0}^{t} G_{0}\left(s, u_{s}, v_{s}\right) \mathrm{d} W(s), t \geq 0 \\
u(0)=u_{0}, \\
u(t)=\varphi_{1}(t), v(t)=\varphi_{2}(t), \text { a.e. } t \in(-h, 0)
\end{array}\right.
$$

where $V$ and $H$ are two real separable Hilbert spaces such that $V \subset H \equiv H^{*} \subset V^{*}$ where the injections are continuous and dense. We denote by $\|\cdot\|,|\cdot|$ and $\|\cdot\|_{*}$ the norms in $V, H$ and 
$V^{*}$ respectively; by $(\cdot, \cdot)$ the inner product in $H$, and by $\langle\cdot, \cdot\rangle$ the duality product between $V^{*}$ and $V$. Let us denote by $c>0$ a constant such that $|x| \leq c\|x\|, \forall x \in V$. We suppose that $\varphi_{1} \in I^{2}(-h, 0 ; V), \varphi_{2} \in I^{2}(-h, 0 ; H), u_{0} \in L^{2}\left(\Omega, \mathcal{F}_{0}, P ; V\right)$ and $v_{0} \in L^{2}\left(\Omega, \mathcal{F}_{0}, P ; H\right)$, and let $A(t): V \rightarrow V^{*}, t \geq 0$, be a family of operators satisfying:

(A.1) $A(t)$ is self-adjoint for each $t \geq 0$.

(A.2) $A(t) \in \mathcal{L}\left(V, V^{*}\right) \forall t \geq 0$, and there exists $c_{A}>0$ such that $\|A(t) u\|_{*} \leq c_{A}\|u\|$, $\forall t \geq 0, \forall u \in V$.

(A.3) $\exists \alpha>0$ such that $\langle A(t) u, u\rangle \geq \alpha\|u\|^{2}, \forall t \geq 0, \forall u \in V$.

$(A .4)\langle A(\cdot) u, \widetilde{u}\rangle \in C^{1}(0,+\infty), \forall u, \widetilde{u} \in V$, and $\left\langle A^{\prime}(t) u, u\right\rangle \leq 0, \forall t \geq 0, \forall u \in V$, where $\left\langle A^{\prime}(t) u, \widetilde{u}\right\rangle$ denotes $\frac{\mathrm{d}}{\mathrm{d} t}\langle A(t) u, \widetilde{u}\rangle$.

(A.5) there exists a Banach space $X$ such that $X \subset\{u \in V ; A(t) u \in H, \forall t \geq 0\}$, the injection of $X$ in $V$ is continuous, and $X$ is dense in $H$.

Let $B(t, \cdot): V \rightarrow V^{*}$ be a family of nonlinear operators defined a.e. $t \geq 0$ and satisfying:

(B.1) $\forall v \in V$, the map $t \in(0,+\infty) \rightarrow B(t, v) \in V^{*}$ is Lebesgue measurable.

(B.2) the map $\theta \in \mathbb{R} \rightarrow\langle B(t, v+\theta w), z\rangle \in \mathbb{R}$ is continuous $\forall v, w, z \in V$, a.e. $t \geq 0$.

(B.3) there exists $c_{B}>0$ such that $\|B(t, v)\|_{*} \leq c_{B}\|v\|, \forall v \in V$, a.e. $t \geq 0$.

(B.4) there exists $\beta>0$ such that $\langle B(t, v)-B(t, \widetilde{v}), v-\widetilde{v}\rangle \geq \beta\|v-\widetilde{v}\|^{2}, \forall v, \widetilde{v} \in V$, a.e. $t \geq 0$.

Let $F_{0}:[0,+\infty) \times C(-h, 0 ; V) \times C(-h, 0 ; H) \rightarrow H$ and $G_{0}:[0,+\infty) \times C(-h, 0 ; V) \times$ $C(-h, 0 ; H) \rightarrow H$ be two families of nonlinear operators defined a.e. $t \geq 0$ such that:

$\left(F_{0} .1\right) \forall(\xi, \eta) \in C(-h, 0 ; V) \times C(-h, 0 ; H)$ the map $t \in(0,+\infty) \rightarrow F_{0}(t, \xi, \eta) \in H$ is Lebesgue measurable, a.e. $t \geq 0$.

$\left(F_{0} .2\right) \quad F_{0}(t, 0,0)=0$, a.e. $t \geq 0$.

$\left(F_{0} .3\right)$ there exist $C_{F_{0}, H}, C_{F_{0}, V}>0$ such that $\forall \xi, \widetilde{\xi} \in C(-h, 0 ; V), \forall \eta, \widetilde{\eta} \in C(-h, 0 ; H)$ and a.e. $t \geq 0$,

$$
\left|F_{0}(t, \xi, \eta)-F_{0}(t, \widetilde{\xi}, \widetilde{\eta})\right|^{2} \leq C_{F_{0}, V}\|\xi-\widetilde{\xi}\|_{C(-h, 0 ; V)}^{2}+C_{F_{0}, H}|\eta-\widetilde{\eta}|_{C(-h, 0 ; H)}^{2}
$$


$\left(F_{0} .4\right)$ there exist $m_{0}>0$ and constants $K_{F_{0}, H}=K_{F_{0}, H}\left(m_{0}, h\right), K_{F_{0}, V}=K_{F_{0}, V}\left(m_{0}, h\right) \geq 0$ such that for all $m \in\left[0, m_{0}\right], \forall x, \widetilde{x} \in C(-h, T ; V), \forall y, \widetilde{y} \in C(-h, T ; H)$, and $\forall t \geq 0$

$$
\begin{aligned}
& \int_{0}^{t} \mathrm{e}^{m s}\left|F_{0}\left(s, x_{s}, y_{s}\right)-F_{0}\left(s, \widetilde{x}_{s}, \widetilde{y}_{s}\right)\right|^{2} \mathrm{~d} s \\
& \leq K_{F_{0}, V} \int_{-h}^{t} \mathrm{e}^{m s}\|x(s)-\widetilde{x}(s)\|^{2} \mathrm{~d} s+K_{F_{0}, H} \int_{-h}^{t} \mathrm{e}^{m s}|y(s)-\widetilde{y}(s)|^{2} \mathrm{~d} s .
\end{aligned}
$$

$\left(G_{0} .1\right) \forall(\xi, \eta) \in C(-h, 0 ; V) \times C(-h, 0 ; H)$ the map $t \in(0,+\infty) \rightarrow G_{0}(t, \xi, \eta) \in H$ is Lebesgue measurable, a.e. $t \geq 0$.

$\left(G_{0} .2\right) G_{0}(t, 0,0)=0$, a.e. $t \geq 0$.

$\left(G_{0} .3\right)$ there exist $C_{G_{0}, H}, C_{G_{0}, V}>0$ such that $\forall \xi, \widetilde{\xi} \in C(-h, 0 ; V), \forall \eta, \widetilde{\eta} \in C(-h, 0 ; H)$ and a.e. $t \geq 0$,

$$
\left|G_{0}(t, \xi, \eta)-G_{0}(t, \widetilde{\xi}, \widetilde{\eta})\right|^{2} \leq C_{G_{0}, V}\|\xi-\widetilde{\xi}\|_{C(-h, 0 ; V)}^{2}+C_{G_{0}, H}|\eta-\widetilde{\eta}|_{C(-h, 0 ; H)}^{2} .
$$

$\left(G_{0} .4\right)$ there exist $m_{0}>0$ and constants $K_{G_{0}, H}=K_{G_{0}, H}\left(m_{0}, h\right), K_{G_{0}, V}=K_{G_{0}, V}\left(m_{0}, h\right) \geq 0$ such that for all $m \in\left[0, m_{0}\right], \forall x, \widetilde{x} \in C(-h, T ; V), \forall y, \widetilde{y} \in C(-h, T ; H)$, and $\forall t \geq 0$

$$
\begin{aligned}
& \int_{0}^{t} \mathrm{e}^{m s}\left|G_{0}\left(s, x_{s}, y_{s}\right)-G_{0}\left(s, \widetilde{x}_{s}, \widetilde{y}_{s}\right)\right|^{2} \mathrm{~d} s \\
& \leq K_{G_{0}, V} \int_{-h}^{t} \mathrm{e}^{m s}\|x(s)-\widetilde{x}(s)\|^{2} \mathrm{~d} s+K_{G_{0}, H} \int_{-h}^{t} \mathrm{e}^{m s}|y(s)-\widetilde{y}(s)|^{2} \mathrm{~d} s .
\end{aligned}
$$

A similar analysis to that in Remark 1 in [8] shows that, under our assumptions all the integrals appearing in problem $(P)$ are well defined, and therefore, the above problem makes sense.

On the other hand, assumptions $\left(F_{0} .2\right),\left(G_{0} .2\right)$ are motivated by our interest in analysing the stability of the zero solution to our problem, but they are not necessary to prove existence of solution. Several results on the existence and uniqueness of solutions for delay stochastic evolution equations of second order in time can be seen in Garrido-Atienza [7], and GarridoAtienza and Real [8]. In particular, we have:

Theorem 1 Assume that hypotheses $(A .1)-(A .5),(B .1)-(B .4),\left(F_{0} .1\right)-\left(F_{0} .4\right),\left(G_{0} .1\right)-$ $\left(G_{0} .4\right)$ hold. Then, if $\varphi_{1} \in I^{2}(-h, 0 ; V), \varphi_{2} \in I^{2}(-h, 0 ; H), u_{0} \in L^{2}\left(\Omega, \mathcal{F}_{0}, P ; V\right)$ and $v_{0} \in L^{2}\left(\Omega, \mathcal{F}_{0}, P ; H\right)$, there exists a unique solution $(u, v)$ to problem $(P)$, for all $T>0$.

We start with a result on the mean square stability for problem $(P)$. 
Theorem 2 Suppose that assumptions $(A .1)-(A .5),(B .1)-(B .4),\left(F_{0} .1\right)-\left(F_{0} .4\right),\left(G_{0} .1\right)-$ $\left(G_{0} .4\right)$ hold. In addition, assume that there exist constants $\varepsilon>0$ and $\delta>0$ such that

$$
\left\{\begin{array}{l}
2 \alpha^{3 / 2} C_{\delta}>c K_{\varepsilon} \\
\text { and } \\
C_{\delta}\left(2 \beta-c^{2}\left(2 K_{F_{0}, H}^{1 / 2}+\varepsilon+K_{G_{0}, H}\right)\right)>\left(4 \alpha^{2} \delta\right)^{-1}\left(4 \alpha \delta c^{2}+\left(c^{2} K_{F_{0}, H}^{1 / 2}+c_{B}\right)^{2}\right) K_{\varepsilon},
\end{array}\right.
$$

where $C_{\delta}=1-\delta-c K_{F_{0}, V}^{1 / 2} \alpha^{-1}$ and $K_{\varepsilon}=K_{F_{0}, V} \varepsilon^{-1}+K_{G_{0}, V}$. Then, the zero solution of problem $(P)$ is exponentially stable in mean square, i.e., there exist $m \in\left(0, m_{0}\right]$ and $K_{1}=K_{1}\left(m_{0}, h\right)>0$ such that, for all $t \geq 0$,

$$
E\left(|v(t)|^{2}+\|u(t)\|^{2}\right) \leq K_{1}\left(E\left|v_{0}\right|^{2}+E\left\|u_{0}\right\|^{2}+\left|\varphi_{2}\right|_{I_{H}^{2}}^{2}+\left\|\varphi_{1}\right\|_{I_{V}^{2}}^{2}\right) e^{-m t},
$$

for any solution $(u, v)$ of $(P)$.

Proof. Let $m \in\left(0, m_{0}\right]$. Applying Itô's formula to the process $\mathrm{e}^{m t}|v(t)|^{2}+\mathrm{e}^{m t}\langle A(t) u(t), u(t)\rangle$ and thanks to $(A .4)$ and $(B .4)$, we obtain for each $t \geq 0$ and $P$-a.s.

$$
\begin{aligned}
& \mathrm{e}^{m t} E|v(t)|^{2}+\mathrm{e}^{m t} E\langle A(t) u(t), u(t)\rangle \\
& \leq E\left|v_{0}\right|^{2}+E\left\langle A(0) u_{0}, u_{0}\right\rangle+m \int_{0}^{t} \mathrm{e}^{m s} E|v(s)|^{2} \mathrm{~d} s \\
& +m \int_{0}^{t} \mathrm{e}^{m s} E\langle A(s) u(s), u(s)\rangle \mathrm{d} s-2 \beta \int_{0}^{t} \mathrm{e}^{m s} E\|v(s)\|^{2} \mathrm{~d} s \\
& +2 \int_{0}^{t} \mathrm{e}^{m s} E\left(F_{0}\left(s, u_{s}, v_{s}\right), v(s)\right) \mathrm{d} s+\int_{0}^{t} \mathrm{e}^{m s} E\left|G_{0}\left(s, u_{s}, v_{s}\right)\right|^{2} \mathrm{~d} s .
\end{aligned}
$$

As $\mathrm{e}^{m t} \leq 1, \forall t \in[-h, 0]$, from $\left(F_{0} .4\right)$ and $(A .3)$ we obtain

$$
\begin{aligned}
& 2 \int_{0}^{t} \mathrm{e}^{m s} E\left(F_{0}\left(s, u_{s}, v_{s}\right), v(s)\right) \mathrm{d} s \\
& \leq 2\left(\int_{0}^{t} \mathrm{e}^{m s} E|v(s)|^{2} \mathrm{~d} s\right)^{1 / 2}\left(\int_{0}^{t} \mathrm{e}^{m s} E\left|F_{0}\left(s, u_{s}, v_{s}\right)\right|^{2} \mathrm{~d} s\right)^{1 / 2} \\
& \leq 2 K_{F_{0}, H}^{1 / 2} \int_{-h}^{t} \mathrm{e}^{m s} E|v(s)|^{2} \mathrm{~d} s+2\left(K_{F_{0}, V} \int_{-h}^{t} \mathrm{e}^{m s} E\|u(s)\|^{2} \mathrm{~d} s\right)^{1 / 2}\left(\int_{0}^{t} \mathrm{e}^{m s} E|v(s)|^{2} \mathrm{~d} s\right)^{1 / 2} \\
& \leq\left(2 K_{F_{0}, H}^{1 / 2}+\varepsilon\right) \int_{0}^{t} \mathrm{e}^{m s} E|v(s)|^{2} \mathrm{~d} s+K_{F_{0}, V}(\alpha \varepsilon)^{-1} \int_{0}^{t} \mathrm{e}^{m s} E\langle A(s) u(s), u(s)\rangle \mathrm{d} s \\
& +K_{F_{0}, V} \varepsilon^{-1}\left\|\varphi_{1}\right\|_{I_{V}^{2}}^{2}+2 K_{F_{0}, H}^{1 / 2}\left|\varphi_{2}\right|_{I_{H}^{2}}^{2},
\end{aligned}
$$

for $\varepsilon>0$, and by $\left(G_{0} .4\right)$ and $(A .3)$,

$$
\begin{aligned}
\int_{0}^{t} \mathrm{e}^{m s} E\left|G_{0}\left(s, u_{s}, v_{s}\right)\right|^{2} \mathrm{~d} s & \leq K_{G_{0}, H}\left|\varphi_{2}\right|_{I_{H}^{2}}^{2}+K_{G_{0}, H} \int_{0}^{t} \mathrm{e}^{m s} E|v(s)|^{2} \mathrm{~d} s \\
& +K_{G_{0}, V}\left\|\varphi_{1}\right\|_{I_{V}^{2}}^{2}+K_{G_{0}, V} \alpha^{-1} \int_{0}^{t} \mathrm{e}^{m s} E\langle A(s) u(s), u(s)\rangle \mathrm{d} s .
\end{aligned}
$$


Thus, if we substitute these inequalities into (4) we have

$$
\begin{aligned}
& \mathrm{e}^{m t} E|v(t)|^{2}+\mathrm{e}^{m t} E\langle A(t) u(t), u(t)\rangle \\
& \leq E\left|v_{0}\right|^{2}+E\left\langle A(0) u_{0}, u_{0}\right\rangle+K_{\varepsilon}\left\|\varphi_{1}\right\|_{I_{V}^{2}}^{2}+\left(2 K_{F_{0}, H}^{1 / 2}+K_{G_{0}, H}\right)\left|\varphi_{2}\right|_{I_{H}^{2}}^{2} \\
& +\left(m+2 K_{F_{0}, H}^{1 / 2}+\varepsilon+K_{G_{0}, H}\right) \int_{0}^{t} \mathrm{e}^{m s} E|v(s)|^{2} \mathrm{~d} s-2 \beta \int_{0}^{t} \mathrm{e}^{m s} E\|v(s)\|^{2} \mathrm{~d} s \\
& +\left(m+K_{\varepsilon} \alpha^{-1}\right) \int_{0}^{t} \mathrm{e}^{m s} E\langle A(s) u(s), u(s)\rangle \mathrm{d} s .
\end{aligned}
$$

Now, we estimate the last integral on the right hand side of (5). From

$$
\begin{aligned}
& \mathrm{d}\left(\mathrm{e}^{m t}(u(t), v(t))\right)=m \mathrm{e}^{m t}(u(t), v(t)) \mathrm{d} t+\mathrm{e}^{m t}|v(t)|^{2} \mathrm{~d} t-\mathrm{e}^{m t}\langle A(t) u(t), u(t)\rangle \mathrm{d} t \\
& -\mathrm{e}^{m t}\langle B(t, v(t)), u(t)\rangle \mathrm{d} t+\mathrm{e}^{m t}\left(F_{0}\left(t, u_{t}, v_{t}\right), u(t)\right)+\mathrm{e}^{m t}\left(G_{0}\left(t, u_{t}, v_{t}\right), u(t)\right) \mathrm{d} W(t),
\end{aligned}
$$

we deduce

$$
\begin{aligned}
& \mathrm{e}^{m t} E(u(t), v(t))=E\left(u_{0}, v_{0}\right)+m \int_{0}^{t} \mathrm{e}^{m s} E(u(s), v(s)) \mathrm{d} s \\
& +\int_{0}^{t} \mathrm{e}^{m s} E|v(s)|^{2} \mathrm{~d} s-\int_{0}^{t} \mathrm{e}^{m s} E\langle A(s) u(s), u(s)\rangle \mathrm{d} s \\
& -\int_{0}^{t} \mathrm{e}^{m s} E\langle B(s, v(s)), u(s)\rangle \mathrm{d} s+\int_{0}^{t} \mathrm{e}^{m s} E\left(F_{0}\left(s, u_{s}, v_{s}\right), u(s)\right) \mathrm{d} s .
\end{aligned}
$$

Consequently,

$$
\begin{aligned}
& \int_{0}^{t} \mathrm{e}^{m s} E\langle A(s) u(s), u(s)\rangle \mathrm{d} s \\
& \leq E\left(u_{0}, v_{0}\right)+\int_{0}^{t} \mathrm{e}^{m s} E|v(s)|^{2} \mathrm{~d} s \\
& +\left(c^{2} m+c_{B}\right)\left(\int_{0}^{t} \mathrm{e}^{m s} E\|v(s)\|^{2} \mathrm{~d} s\right)^{1 / 2}\left(\alpha^{-1} \int_{0}^{t} \mathrm{e}^{m s} E\langle A(s) u(s), u(s)\rangle \mathrm{d} s\right)^{1 / 2} \\
& +\left(\int_{0}^{t} \mathrm{e}^{m s} E\left|F_{0}\left(s, u_{s}, v_{s}\right)\right|^{2} \mathrm{~d} s\right)^{1 / 2}\left(c^{2} \int_{0}^{t} \mathrm{e}^{m s} E\|u(s)\|^{2} \mathrm{~d} s\right)^{1 / 2} \\
& +\left(\mathrm{e}^{m t} E|v(t)|^{2}\right)^{1 / 2}\left(c^{2} \alpha^{-1} \mathrm{e}^{m t} E\langle A(t) u(t), u(t)\rangle\right)^{1 / 2} \\
& \leq E\left(u_{0}, v_{0}\right)+\int_{0}^{t} \mathrm{e}^{m s} E|v(s)|^{2} \mathrm{~d} s+c K_{F_{0}, V}^{1 / 2}\left\|\varphi_{1}\right\|_{I_{V}^{2}}^{2}+\left(c^{2}\left(m+K_{F_{0}, H}^{1 / 2}\right)+c_{B}\right)^{2}(4 \alpha \delta)^{-1}\left|\varphi_{2}\right|_{I_{H}^{2}}^{2} \\
& +\left(c^{2}\left(m+K_{F_{0}, H}^{1 / 2}\right)+c_{B}\right)^{2}(4 \alpha \delta)^{-1} \int_{0}^{t} \mathrm{e}^{m s} E\|v(s)\|^{2} \mathrm{~d} s \\
& +\left(\delta+c K_{F_{0}, V}^{1 / 2} \alpha^{-1}\right) \int_{0}^{t} \mathrm{e}^{m s} E\langle A(s) u(s), u(s)\rangle \mathrm{d} s \\
& +c\left(2 \alpha^{1 / 2}\right)^{-1} \mathrm{e}^{m t} E\left(|v(t)|^{2}+\langle A(t) u(t), u(t)\rangle\right)
\end{aligned}
$$


and thus

$$
\begin{aligned}
& C_{\delta} \int_{0}^{t} \mathrm{e}^{m s} E\langle A(s) u(s), u(s)\rangle \mathrm{d} s \\
& \leq E\left(u_{0}, v_{0}\right)+c K_{F_{0}, V}^{1 / 2}\left\|\varphi_{1}\right\|_{I_{V}^{2}}^{2}+\left(c^{2}\left(m+K_{F_{0}, H}^{1 / 2}\right)+c_{B}\right)^{2}(4 \alpha \delta)^{-1}\left|\varphi_{2}\right|_{I_{H}^{2}}^{2} \\
& +c\left(2 \alpha^{1 / 2}\right)^{-1} \mathrm{e}^{m t} E\left(|v(t)|^{2}+\langle A(t) u(t), u(t)\rangle\right) \\
& +(4 \alpha \delta)^{-1}\left(4 \alpha \delta c^{2}+\left(c^{2}\left(m+K_{F_{0}, H}^{1 / 2}\right)+c_{B}\right)^{2}\right) \int_{0}^{t} \mathrm{e}^{m s} E\|v(s)\|^{2} \mathrm{~d} s .
\end{aligned}
$$

Due to the first condition in (2), the constant $C_{\delta}$ is positive. Therefore, we have

$$
\begin{aligned}
& \left(1-c\left(m+K_{\varepsilon} \alpha^{-1}\right)\left(2 \alpha^{1 / 2} C_{\delta}\right)^{-1}\right) \mathrm{e}^{m t} E\left(|v(t)|^{2}+\langle A(t) u(t), u(t)\rangle\right) \\
& \leq E\left|v_{0}\right|^{2}+E\left\langle A(0) u_{0}, u_{0}\right\rangle+\left(m+\alpha^{-1} K_{\varepsilon}\right) C_{\delta}^{-1} E\left(u_{0}, v_{0}\right) \\
& +\left(K_{\varepsilon}+c\left(m+K_{\varepsilon} \alpha^{-1}\right) K_{F_{0}, V}^{1 / 2} C_{\delta}^{-1}\right)\left\|\varphi_{1}\right\|_{I_{V}^{2}}^{2} \\
& +\left(2 K_{F_{0}, H}^{1 / 2}+K_{G_{0}, H}+\left(c^{2}\left(m+K_{F_{0}, H}^{1 / 2}\right)+c_{B}\right)^{2}\left(m+K_{\varepsilon} \alpha^{-1}\right)\left(4 \alpha \delta C_{\delta}\right)^{-1}\right)\left|\varphi_{2}\right|_{I_{H}^{2}}^{2} \\
& +\left(c^{2}\left(m+2 K_{F_{0}, H}^{1 / 2}+\varepsilon+K_{G_{0}, H}\right)-2 \beta\right) \int_{0}^{t} \mathrm{e}^{m s} E\|v(s)\|^{2} \mathrm{~d} s \\
& +\left(m+K_{\varepsilon} \alpha^{-1}\right)\left(4 \alpha \delta C_{\delta}\right)^{-1}\left(4 \alpha \delta c^{2}+\left(c^{2}\left(m+K_{F_{0}, H}^{1 / 2}\right)+c_{B}\right)^{2}\right) \int_{0}^{t} \mathrm{e}^{m s} E\|v(s)\|^{2} \mathrm{~d} s .
\end{aligned}
$$

Finally, taking into account the expressions of $C_{\delta}$ and $K_{\varepsilon}$, Eq. (2) implies, for $m>0$ small enough, that

$$
\begin{aligned}
0< & 1-c\left(m+K_{\varepsilon} \alpha^{-1}\right)\left(2 \alpha^{1 / 2} C_{\delta}\right)^{-1}, \\
2 \beta> & c^{2}\left(m+2 K_{F_{0}, H}^{1 / 2}+\varepsilon+K_{G_{0}, H}\right) \\
& +\left(m+K_{\varepsilon} \alpha^{-1}\right)\left(4 \alpha \delta C_{\delta}\right)^{-1}\left(4 \alpha \delta c^{2}+\left(c^{2}\left(m+K_{F_{0}, H}^{1 / 2}\right)+c_{B}\right)^{2}\right),
\end{aligned}
$$

which together with $(A .2)$ and (A.3) finishes the proof.

Remark 3 Observe that condition (2) can be rewritten in an easier way in some particular cases. Indeed, if we assume that $K_{F_{0}, V}=0$ and fix $\delta=1 / 2$ in Theorem 2, then (2) holds if we suppose

$$
\left\{\begin{array}{l}
K_{G_{0}, V}<c^{-1} \alpha^{3 / 2} \\
K_{G_{0}, H}<2 \beta c^{-2}-2\left(\alpha+\left(c K_{F_{0}, H}^{1 / 2}+c_{B} c^{-1}\right)^{2}\right) \alpha^{-2} K_{G_{0}, V}-2 K_{F_{0}, H}^{1 / 2} .
\end{array}\right.
$$

For our functional problem $(P)$, we will prove that the sample paths tend to zero exponentially fast as $t \rightarrow \infty$ whenever the exponential mean square stability holds, in particular, under the assumptions of Theorem 2. To this end, we will use a technique (based on the 
Burkholder-Davis-Gundy inequality, the Doob inequality and the Borel-Cantelli lemma) which has proven very fruitful for parabolic equations (see, e.g. [1],[12],[13]).

First, we need the following result:

Lemma 4 In the conditions of Theorem 2, there exist constants $C_{1}, C_{2}>0$ such that for any solution $(u, v)$ of problem $(P)$ it follows

$$
\begin{gathered}
\int_{0}^{t} e^{m s} E|v(s)|^{2} d s \leq C_{1}\left(E\left|v_{0}\right|^{2}+E\left\|u_{0}\right\|^{2}+\left|\varphi_{2}\right|_{I_{H}^{2}}^{2}+\left\|\varphi_{1}\right\|_{I_{V}^{2}}^{2}\right), \\
\int_{0}^{t} e^{m s} E\|u(s)\|^{2} d s \leq C_{2}\left(E\left|v_{0}\right|^{2}+E\left\|u_{0}\right\|^{2}+\left|\varphi_{2}\right|_{I_{H}^{2}}^{2}+\left\|\varphi_{1}\right\|_{I_{V}^{2}}^{2}\right),
\end{gathered}
$$

for each $t \geq 0$.

Proof. We first re-write (7) as

$$
\begin{aligned}
& \left(1-c\left(m+K_{\varepsilon} \alpha^{-1}\right)\left(2 \alpha^{1 / 2} C_{\delta}\right)^{-1}\right) \mathrm{e}^{m t} E\left(|v(t)|^{2}+\langle A(t) u(t), u(t)\rangle\right) \\
& \leq \widetilde{C}_{1}\left(E\left|v_{0}\right|^{2}+E\left\|u_{0}\right\|^{2}+\left|\varphi_{2}\right|_{I_{H}^{2}}^{2}+\left\|\varphi_{1}\right\|_{I_{V}^{2}}^{2}\right) \\
& +\left(c^{2}\left(m+2 K_{F_{0}, H}^{1 / 2}+\varepsilon+K_{G_{0}, H}\right)-2 \beta\right) \int_{0}^{t} \mathrm{e}^{m s} E\|v(s)\|^{2} \mathrm{~d} s \\
& +\left(m+K_{\varepsilon} \alpha^{-1}\right)\left(4 \alpha \delta C_{\delta}\right)^{-1}\left(4 \alpha \delta c^{2}+\left(c^{2}\left(m+K_{F_{0}, H}^{1 / 2}\right)+c_{B}\right)^{2}\right) \int_{0}^{t} \mathrm{e}^{m s} E\|v(s)\|^{2} \mathrm{~d} s .
\end{aligned}
$$

Thus, owing to Theorem 2, we can choose $m>0$ such that the constant $\widetilde{C}_{2}$ multiplying $\int_{0}^{t} \mathrm{e}^{m s} E\|v(s)\|^{2} \mathrm{~d} s$ in (7) becomes negative, and hence

$$
\int_{0}^{t} e^{m s} E\|v(s)\|^{2} d s \leq-\widetilde{C}_{1} \widetilde{C}_{2}^{-1}\left(E\left|v_{0}\right|^{2}+E\left\|u_{0}\right\|^{2}+\left|\varphi_{2}\right|_{I_{H}^{2}}^{2}+\left\|\varphi_{1}\right\|_{I_{V}^{2}}^{2}\right) .
$$

Now, to check (9) it is enough to take into account (11) and that $|v|^{2} \leq c^{2}\|v\|^{2}$, for all $v \in V$.

On the other hand, (10) follows from (6) by taking into account (A.2), (A.3), (3) and (11).

Now, using this lemma, we discuss the almost sure stability of solutions to $(P)$.

Theorem 5 Under assumptions in Theorem 2, there exist $K_{2}=K_{2}(m, h), \gamma>0$, and a subset $\Omega_{0} \subset \Omega$ with $P\left(\Omega_{0}\right)=0$ such that, for each $\omega \notin \Omega_{0}$, there exists a positive random variable $T(\omega)$ such that if $(u, v)$ is a solution of $(P)$, it holds

$$
|v(t)|^{2}+\|u(t)\|^{2} \leq K_{2}\left(E\left|v_{0}\right|^{2}+E\left\|u_{0}\right\|^{2}+\left|\varphi_{2}\right|_{I_{H}^{2}}^{2}+\left\|\varphi_{1}\right\|_{I_{V}^{2}}^{2}\right) e^{-\gamma t}, \forall t \geq T(\omega),
$$

i.e., $(u(t), v(t))$ decays exponentially to zero almost surely. 
Proof. We first prove that there exists a positive constant $C$ such that for any $N \in \mathbb{N}$

$$
E\left[\sup _{N \leq t \leq N+1}\left(|v(t)|^{2}+\|u(t)\|^{2}\right)\right] \leq C e^{-m N}\left(E\left|v_{0}\right|^{2}+E\left\|u_{0}\right\|^{2}+\left|\varphi_{2}\right|_{I_{H}^{2}}^{2}+\left\|\varphi_{1}\right\|_{I_{V}^{2}}^{2}\right) .
$$

Indeed, Ito's formula implies once again

$$
\begin{aligned}
& |v(t)|^{2}+\langle A(t) u(t), u(t)\rangle \\
& =|v(N)|^{2}+\langle A(N) u(N), u(N)\rangle+\int_{N}^{t}\left\langle A^{\prime}(s) u(s), u(s)\right\rangle \mathrm{d} s \\
& -2 \int_{N}^{t}\langle B(s, v(s)), v(s)\rangle \mathrm{d} s+2 \int_{N}^{t}\left(F_{0}\left(s, u_{s}, v_{s}\right), v(s)\right) \mathrm{d} s \\
& +\int_{N}^{t}\left|G_{0}\left(s, u_{s}, v_{s}\right)\right|^{2} \mathrm{~d} s+2 \int_{N}^{t}\left(G_{0}\left(s, u_{s}, v_{s}\right), v(s)\right) \mathrm{d} W(s) .
\end{aligned}
$$

Now, $(A .2),(A .3),(A .4)$ and $(B .4)$, yield that

$$
\begin{aligned}
& \sup _{N \leq t \leq N+1}\left(|v(t)|^{2}+\alpha\|u(t)\|^{2}\right) \\
& \leq 2|v(N)|^{2}+2 c_{A}\|u(N)\|^{2}+2 \int_{N}^{N+1}\left(F_{0}\left(s, u_{s}, v_{s}\right), v(s)\right) \mathrm{d} s \\
& +2 \int_{N}^{N+1}\left|G_{0}\left(s, u_{s}, v_{s}\right)\right|^{2} \mathrm{~d} s+4 \sup _{N \leq t \leq N+1}\left(\left|\int_{N}^{t}\left(G_{0}\left(s, u_{s}, v_{s}\right), v(s)\right) \mathrm{d} W(s)\right|\right) .
\end{aligned}
$$

From $\left(F_{0} .4\right),(9)$ and $(10)$, it follows

$$
\begin{aligned}
& 2 \int_{N}^{N+1} E\left(F_{0}\left(s, u_{s}, v_{s}\right), v(s)\right) \mathrm{d} s \\
& \leq 2 \int_{N}^{N+1} \mathrm{e}^{m(s-N)} E\left(F_{0}\left(s, u_{s}, v_{s}\right), v(s)\right) \mathrm{d} s \\
& \leq \mathrm{e}^{-m N}\left(\varepsilon+2 K_{F_{0}, H}^{1 / 2}\right) \int_{0}^{N+1} \mathrm{e}^{m s} E|v(s)|^{2} \mathrm{~d} s+2 \mathrm{e}^{-m N} K_{F_{0}, H}^{1 / 2}\left|\varphi_{2}\right|_{I_{H}^{2}}^{2} \\
& +\mathrm{e}^{-m N} K_{F_{0}, V} \varepsilon^{-1} \int_{0}^{N+1} \mathrm{e}^{m s} E\|u(s)\|^{2} \mathrm{~d} s+\mathrm{e}^{-m N} K_{F_{0}, V} \varepsilon^{-1}\left\|\varphi_{1}\right\|_{I_{V}^{2}}^{2} \\
& \leq \mathrm{e}^{-m N}\left(\varepsilon+2 K_{F_{0}, H}^{1 / 2}\right) C_{1}\left(E\left|v_{0}\right|^{2}+E\left\|u_{0}\right\|^{2}+\left|\varphi_{2}\right|_{I_{H}^{2}}^{2}+\left\|\varphi_{1}\right\|_{I_{V}^{2}}^{2}\right) \\
& +\mathrm{e}^{-m N} K_{F_{0}, V} \varepsilon^{-1} C_{2}\left(E\left|v_{0}\right|^{2}+E\left\|u_{0}\right\|^{2}+\left|\varphi_{2}\right|_{I_{H}^{2}}^{2}+\left\|\varphi_{1}\right\|_{I_{V}^{2}}^{2}\right) \\
& +2 \mathrm{e}^{-m N} K_{F_{0}, H}^{1 / 2}\left|\varphi_{2}\right|_{I_{H}^{2}}^{2}+\mathrm{e}^{-m N} K_{F_{0}, V} \varepsilon^{-1}\left\|\varphi_{1}\right\|_{I_{V}^{2}}^{2} .
\end{aligned}
$$


On the other hand, Burkholder-Davis-Gundy's inequality, $\left(G_{0} \cdot 4\right)$, (9), and (10) imply

$$
\begin{aligned}
& 4 E\left[\sup _{N \leq t \leq N+1}\left(\left|\int_{N}^{t}\left(G_{0}\left(s, u_{s}, v_{s}\right), v(s)\right) \mathrm{d} W(s)\right|\right)\right] \\
& \leq 24 E\left[\left(\sup _{N \leq t \leq N+1}|v(t)|^{2}\right)^{1 / 2}\left(\int_{N}^{N+1}\left|G_{0}\left(s, u_{s}, v_{s}\right)\right|^{2} \mathrm{~d} s\right)^{1 / 2}\right] \\
& \leq \frac{1}{2} E \sup _{N \leq t \leq N+1}|v(t)|^{2}+288 \int_{N}^{N+1} E\left|G_{0}\left(s, u_{s}, v_{s}\right)\right|^{2} \mathrm{~d} s
\end{aligned}
$$

and

$$
\begin{aligned}
& \int_{N}^{N+1} E\left|G_{0}\left(s, u_{s}, v_{s}\right)\right|^{2} \mathrm{~d} s \\
& \leq \int_{N}^{N+1} \mathrm{e}^{m(s-N)} E\left|G_{0}\left(s, u_{s}, v_{s}\right)\right|^{2} \mathrm{~d} s \\
& \leq \mathrm{e}^{-m N}\left(K_{G_{0}, V}\left(1+C_{2}\right)+K_{G_{0}, H}\left(1+C_{1}\right)\right)\left(E\left|v_{0}\right|^{2}+E\left\|u_{0}\right\|^{2}+\left|\varphi_{2}\right|_{I_{H}^{2}}^{2}+\left\|\varphi_{1}\right\|_{I_{V}^{2}}^{2}\right) .
\end{aligned}
$$

Finally, from (3) we have

$$
E\left(|v(N)|^{2}+\|u(N)\|^{2}\right) \leq K_{1}\left(E\left|v_{0}\right|^{2}+E\left\|u_{0}\right\|^{2}+\left|\varphi_{2}\right|_{I_{H}^{2}}^{2}+\left\|\varphi_{1}\right\|_{I_{V}^{2}}^{2}\right) \mathrm{e}^{-m N},
$$

for $t>N$. Then (12) follows from the previous estimates.

Now, given $\varepsilon>0$, the Doob inequality implies

$$
\begin{aligned}
& P\left[\sup _{N \leq t \leq N+1}\left(|v(t)|^{2}+\|u(t)\|^{2}\right) \geq e^{-(m+\varepsilon) N}\right] \\
& \leq e^{(m-\varepsilon) N} E\left[\sup _{N \leq t \leq N+1}\left(|v(t)|^{2}+\|u(t)\|^{2}\right)\right] \\
& \leq C e^{-\varepsilon N}\left(E\left|v_{0}\right|^{2}+E\left\|u_{0}\right\|^{2}+\left|\varphi_{2}\right|_{I_{H}^{2}}^{2}+\left\|\varphi_{1}\right\|_{I_{V}^{2}}^{2}\right)
\end{aligned}
$$

and the Borel-Cantelli lemma can be now applied to complete the proof.

The previous results have been established in [3] in the case in which a more particular family of nonlinear operators $B(t, \cdot)$ appears. Indeed, in that paper we have considered that $B(t, \cdot): H \rightarrow H$ is a family of nonlinear operators defined a.e. $t \geq 0$ and satisfying:

(B.1) $\forall v \in H$, the map $t \in(0,+\infty) \rightarrow B(t, v) \in H$ is Lebesgue measurable.

(B.2) the map $\theta \in \mathbb{R} \rightarrow(B(t, v+\theta w), z) \in \mathbb{R}$ is continuous $\forall v, w, z \in H$, a.e. $t \geq 0$.

(B.3) there exists $c_{B}>0$ such that $|B(t, v)| \leq c_{B}|v|, \forall v \in H$, a.e. $t \geq 0$.

(B.4) there exists $\beta>0$ such that $(B(t, v)-B(t, \widetilde{v}), v-\widetilde{v}) \geq \beta|v-\widetilde{v}|^{2}, \forall v, \widetilde{v} \in H$, a.e. $t \geq 0$. 
Then, we can prove in the same way as in Theorem 2, that the null solution to corresponding problem $(P)$ is exponentially stable in mean square if we suppose that there exist some constants $\varepsilon>0$ and $\delta>0$ such that

$$
\left\{\begin{array}{l}
2 \alpha^{3 / 2} C_{\delta}>c K_{\varepsilon}, \\
\text { and } \\
C_{\delta}\left(2 \beta-\left(2 K_{F_{0}, H}^{1 / 2}+\varepsilon+K_{G_{0}, H}\right)\right)>\left(4 \alpha^{2} \delta\right)^{-1}\left(4 \alpha \delta+c^{2}\left(K_{F_{0}, H}^{1 / 2}+c_{B}\right)^{2}\right) K_{\varepsilon},
\end{array}\right.
$$

If, in addition, we suppose as a particular situation, that $F_{0} \equiv 0, G_{0}(., u ., v)=.G_{0}(., u$. (which means that $K_{G_{0}, H}=0$ ) and we fix $\delta=\frac{1}{2}$, then the null solution to the corresponding problem is exponentially stable in mean square if

$$
K_{G_{0}, V}<\min \left\{c^{-1}, 2 \beta \alpha^{1 / 2}\left(2 \alpha+\left(c c_{B}\right)^{2}\right)^{-1}\right\} \alpha^{3 / 2}
$$

\section{Spatial derivatives in the delayed operators}

This section is devoted to establish analogous asymptotic stability results for a more general delayed stochastic problem of second order in time, that is, the objective is to point out that a theory similar to the one developed in the preceding section can be carry out also by using the same techniques, for the below problem, in which it is accepted that delay can appear in operators containing first order spatial partial derivatives. First of all, we are going to formulate the hypotheses for the new operators:

Let $F_{1}:[0,+\infty) \times C(-h, 0 ; V) \times C(-h, 0 ; H) \rightarrow V^{*}, G_{1}:[0,+\infty) \times C(-h, 0 ; V) \times$ $C(-h, 0 ; V) \rightarrow H$ be two nonlinear operators such that:

$\left(F_{1} .1\right) \forall(\xi, \eta) \in C(-h, 0 ; V) \times C(-h, 0 ; H)$ the map $t \in(0,+\infty) \rightarrow F_{1}(t, \xi, \eta) \in V^{*}$ is Lebesgue measurable, a.e. $t$.

$\left(F_{1} .2\right) \quad F_{1}(t, 0,0)=0$, a.e. $t \geq 0$.

$\left(F_{1} .3\right)$ there exist $C_{F_{1}, H}, C_{F_{1}, V}>0$ such that $\forall \xi, \widetilde{\xi} \in C(-h, 0 ; V), \forall \eta, \widetilde{\eta} \in C(-h, 0 ; H)$ and a.e. $t$,

$$
\left\|F_{1}(t, \xi, \eta)-F_{1}(t, \widetilde{\xi}, \widetilde{\eta})\right\|_{*}^{2} \leq C_{F_{1}, V}\|\xi-\widetilde{\xi}\|_{C(-h, 0 ; V)}^{2}+C_{F_{1}, H}|\eta-\widetilde{\eta}|_{C(-h, 0 ; H)}^{2},
$$

$\left(F_{1} .4\right)$ there exist $m_{0}>0$ and constants $K_{F_{1}, H}=K_{F_{1}, H}\left(m_{0}, h\right), K_{F_{1}, V}=K_{F_{1}, V}\left(m_{0}, h\right) \geq 0$ 
such that for all $m \in\left[0, m_{0}\right], \forall x, \widetilde{x} \in C(-h, T ; V), \forall y, \widetilde{y} \in C(-h, T ; H)$, and $\forall t \geq 0$

$$
\begin{aligned}
& \int_{0}^{t} \mathrm{e}^{m s}\left\|F_{1}\left(s, x_{s}, y_{s}\right)-F_{1}\left(s, \widetilde{x}_{s}, \widetilde{y}_{s}\right)\right\|_{*}^{2} \mathrm{~d} s \\
& \leq K_{F_{1}, V} \int_{-h}^{t} \mathrm{e}^{m s}\|x(s)-\widetilde{x}(s)\|^{2} \mathrm{~d} s+K_{F_{1}, H} \int_{-h}^{t} \mathrm{e}^{m s}|y(s)-\widetilde{y}(s)|^{2} \mathrm{~d} s .
\end{aligned}
$$

$\left(G_{1} .1\right) \forall(\xi, \eta) \in C(-h, 0 ; V) \times C(-h, 0 ; V)$ the map $t \in(0,+\infty) \rightarrow G_{1}(t, \xi, \eta) \in H$ is Lebesgue measurable, a.e. $t$.

$\left(G_{1} .2\right) G_{1}(t, 0,0)=0$, a.e. $t \geq 0$.

$\left(G_{1} .3\right)$ there exist $C_{G_{1}, V}, \widetilde{C}_{G_{1}, V}>0$ such that $\forall \xi, \widetilde{\xi}, \eta, \widetilde{\eta} \in C(-h, 0 ; V)$ and a.e. $t$,

$$
\left|G_{1}(t, \xi, \eta)-G_{1}(t, \widetilde{\xi}, \widetilde{\eta})\right|^{2} \leq C_{G_{1}, V}\|\xi-\widetilde{\xi}\|_{C(-h, 0 ; V)}^{2}+\widetilde{C}_{G_{1}, V}\|\eta-\widetilde{\eta}\|_{C(-h, 0 ; V)}^{2} .
$$

$\left(G_{1} .4\right)$ there exist $m_{0}>0$ and constants $\widetilde{K}_{G_{1}, V}=\widetilde{K}_{G_{1}, V}\left(m_{0}, h\right), K_{G_{1}, V}=K_{G_{1}, V}\left(m_{0}, h\right) \geq 0$ such that $\forall x, \widetilde{x}, y, \widetilde{y} \in C(-h, T ; V)$ and $\forall t \geq 0$,

$$
\begin{aligned}
& \int_{0}^{t} \mathrm{e}^{m s}\left|G_{1}\left(s, x_{s}, y_{s}\right)-G_{1}\left(s, \widetilde{x}_{s}, \widetilde{y}_{s}\right)\right|^{2} \mathrm{~d} s \\
\leq & K_{G_{1}, V} \int_{-h}^{t} \mathrm{e}^{m s}\|x(s)-\widetilde{x}(s)\|^{2} \mathrm{~d} s+\widetilde{K}_{G_{1}, V} \int_{-h}^{t} \mathrm{e}^{m s}\|y(s)-\widetilde{y}(s)\|^{2} \mathrm{~d} s .
\end{aligned}
$$

In this case, we consider the following problem

$$
\left\{\begin{array}{l}
u \in I^{2}(-h, T ; V) \cap L^{2}(\Omega ; C(0, T ; V)), \text { for all } T>0 \\
v \in I^{2}(-h, T ; V) \cap L^{2}(\Omega ; C(0, T ; H)), \text { for all } T>0 \\
u^{\prime}(t)=v(t), t \in[0, T] \\
v(t)+\int_{0}^{t} A(s) u(s) \mathrm{d} s+\int_{0}^{t} B(s, v(s)) \mathrm{d} s \\
=v_{0}+\int_{0}^{t}\left(F_{0}\left(s, u_{s}, v_{s}\right)+F_{1}\left(s, u_{s}, v_{s}\right)\right) \mathrm{d} s \\
+\int_{0}^{t}\left(G_{0}\left(s, u_{s}, v_{s}\right)+G_{1}\left(s, u_{s}, v_{s}\right)\right) \mathrm{d} W(s), t \in[0, T], \\
u(0)=u_{0}, \\
u(t)=\varphi_{1}(t), v(t)=\varphi_{2}(t), \text { a.e. } t \in(-h, 0),
\end{array}\right.
$$

where $\varphi_{1}, \varphi_{2} \in I^{2}(-h, 0 ; V), u_{0} \in L^{2}\left(\Omega, \mathcal{F}_{0}, P ; V\right), v_{0} \in L^{2}\left(\Omega, \mathcal{F}_{0}, P ; H\right)$ are given.

As is was pointed out in the previous section, all the integrals in problem $(Q)$ are well defined.

Results about the existence and uniqueness of solutions for $(Q)$ can be found in GarridoAtienza and Real [8]. In particular, for our problem $(Q)$ in the mentionated work it is necessary the additional hypotheses: 
(H) $\exists \lambda>0, m, \widehat{m} \geq 0$ such that for $x, \widetilde{x}, y, \widetilde{y} \in L^{2}(-h, T ; V)$ it holds, $\forall t \geq 0$,

$$
\begin{aligned}
& 2 \int_{0}^{t} \mathrm{e}^{m s}\langle B(s, y(s))-B(s, \widetilde{y}(s)), y(s)-\widetilde{y}(s)\rangle \mathrm{d} s \\
& +\widehat{m} \int_{-h}^{0} \mathrm{e}^{m s}\left(\|x(s)-\widetilde{x}(s)\|^{2}+\|y(s)-\widetilde{y}(s)\|^{2}\right) \mathrm{d} s \\
\geq & \lambda \int_{0}^{t} \mathrm{e}^{m s}\|y(s)-\widetilde{y}(s)\|^{2} \mathrm{~d} s+\int_{0}^{t} \mathrm{e}^{m s}\left|G_{1}\left(s, x_{s}, y_{s}\right)-G_{1}\left(s, \widetilde{x}_{s}, \widetilde{y}_{s}\right)\right|^{2} \mathrm{~d} s .
\end{aligned}
$$

Next, we will check that, under the previous assumptions, a similar result to Theorem 2 holds true. In the proof, we will suppose that the term $F_{0} \equiv 0$, otherwise we can consider a new operator $\widehat{F}_{1}:[0,+\infty) \times C(-h, 0 ; V) \times C(-h, 0 ; H) \rightarrow V^{*}$ defined by $\widehat{F}_{1}=F_{0}+F_{1}$. Also, for the sake of clarity, we will assume that $\widetilde{K}_{G_{1}, V}=K_{G_{1}, V} \triangleq K_{G_{1}}$.

Theorem 6 Supose that assumptions $(A .1)-(A .5),(B .1)-(B .5),\left(F_{1} .1\right)-\left(F_{1} .4\right),\left(G_{0} .1\right)-$ $\left(G_{0} .4\right),\left(G_{1} .1\right)-\left(G_{1} .4\right)$ and $(H)$ hold. In addition, assume that there exist $\delta>0$ and $\lambda>0$ such that

$$
\left\{\begin{array}{l}
2 \alpha^{3 / 2} C_{\delta}>c K_{\lambda}, \\
\text { and } \\
C_{\delta}\left(\frac{\lambda}{3}-c^{2}\left(\frac{3 K_{F_{1}, H}}{\lambda}+K_{G_{0}, H}+\frac{3 K_{G_{1}}}{\lambda} K_{G_{0}, H}\right)\right) \\
>\left(2 \alpha^{2} \delta\right)^{-1}\left(2 \alpha \delta c^{2}+c^{2} K_{F_{1}, H}+c_{B}{ }^{2}\right) K_{\lambda},
\end{array}\right.
$$

where $C_{\delta}=1-\delta-K_{F_{1}, V}^{1 / 2} \alpha^{-1}$ and $K_{\lambda}=\frac{\lambda}{3}+\frac{3 K_{G_{1}}}{\lambda} K_{G_{0}, V}+K_{G_{0}, V}+\frac{3 K_{F_{1}, V}}{\lambda}$. Then the zero solution of problem $(Q)$ is exponentially stable in mean square.

Proof. Let $m$ be a positive constant. Arguing as in the proof of Theorem 2, but taking now into account the new hypotheses on operators $F_{1}$ and $G_{1},(H)$ and the expression of $K_{\lambda}$, we have

$$
\begin{aligned}
& \mathrm{e}^{m t} E|v(t)|^{2}+\mathrm{e}^{m t} E\langle A(t) u(t), u(t)\rangle+\frac{\lambda}{3} \int_{0}^{t} \mathrm{e}^{m s} E\|v(s)\|^{2} \mathrm{~d} s \\
& \leq E\left|v_{0}\right|^{2}+E\left\langle A(0) u_{0}, u_{0}\right\rangle+\left(K_{\lambda}+\widehat{m}\right)\left\|\varphi_{1}\right\|_{I_{V}^{2}}^{2}+\left(\frac{\lambda}{3}+\widehat{m}\right)\left\|\varphi_{2}\right\|_{I_{V}^{2}}^{2} \\
& +\left(K_{G_{0}, H}+\frac{3 K_{G_{1}}}{\lambda} K_{G_{0}, H}+\frac{3 K_{F_{1}, H}}{\lambda}\right)\left|\varphi_{2}\right|_{I_{H}^{2}}^{2} \\
& +\left(m+\frac{3 K_{F_{1}, H}}{\lambda}+K_{G_{0}, H}+\frac{3 K_{G_{1}}}{\lambda} K_{G_{0}, H}\right) \int_{0}^{t} \mathrm{e}^{m s} E|v(s)|^{2} \mathrm{~d} s \\
& +\left(m+K_{\lambda} \alpha^{-1}\right) \int_{0}^{t} \mathrm{e}^{m s} E\langle A(s) u(s), u(s)\rangle \mathrm{d} s .
\end{aligned}
$$


Using the expression of $\mathrm{e}^{m t} E(u(t), v(t))$, we have

$$
\begin{aligned}
& \int_{0}^{t} \mathrm{e}^{m s} E\langle A(s) u(s), u(s)\rangle \mathrm{d} s \\
& \leq E\left(u_{0}, v_{0}\right)+\int_{0}^{t} \mathrm{e}^{m s} E|v(s)|^{2} \mathrm{~d} s+K_{F_{1}, V}^{1 / 2}\left\|\varphi_{1}\right\|_{I_{V}^{2}}^{2}+\left(m c+K_{F_{1}, H}^{1 / 2}\right)^{2}(2 \alpha \delta)^{-1}\left|\varphi_{2}\right|_{I_{H}^{2}}^{2} \\
& +c\left(2 \alpha^{1 / 2}\right)^{-1} \mathrm{e}^{m t} E\left(|v(t)|^{2}+\langle A(t) u(t), u(t)\rangle\right)+\left(m c+K_{F_{1}, H}^{1 / 2}\right)^{2}(2 \alpha \delta)^{-1} \int_{0}^{t} \mathrm{e}^{m s} E|v(s)|^{2} \mathrm{~d} s \\
& +\left(\delta+K_{F_{1}, V}^{1 / 2} \alpha^{-1}\right) \int_{0}^{t} \mathrm{e}^{m s} E\langle A(s) u(s), u(s)\rangle \mathrm{d} s+c_{B}^{2}(2 \alpha \delta)^{-1} \int_{0}^{t} \mathrm{e}^{m s} E\|v(s)\|^{2} \mathrm{~d} s
\end{aligned}
$$

and then, it follows

$$
\begin{aligned}
& \left(1-c\left(m+K_{\lambda} \alpha^{-1}\right)\left(2 \alpha^{1 / 2} C_{\delta}\right)^{-1}\right) \mathrm{e}^{m t} E\left(|v(t)|^{2}+\langle A(t) u(t), u(t)\rangle\right) \\
& +\frac{\lambda}{3} \int_{0}^{t} \mathrm{e}^{m s} E\|v(s)\|^{2} \mathrm{~d} s \\
& \leq E\left|v_{0}\right|^{2}+E\left\langle A(0) u_{0}, u_{0}\right\rangle+\left(m+\alpha^{-1} K_{\lambda}\right) C_{\delta}^{-1} E\left(u_{0}, v_{0}\right) \\
& +\left(K_{\lambda}+\widehat{m}+\left(m+K_{\lambda} \alpha^{-1}\right) K_{F_{1}, V}^{1 / 2} C_{\delta}^{-1}\right)\left\|\varphi_{1}\right\|_{I_{V}^{2}}^{2}+\left(\frac{\lambda}{3}+\widehat{m}\right)\left\|\varphi_{2}\right\|_{I_{V}^{2}}^{2} \\
& +\left(K_{G_{0}, H}+\frac{3 K_{G_{1}}}{\lambda} K_{G_{0}, H}+\frac{3 K_{F_{1}, H}}{\lambda}\right)\left|\varphi_{2}\right|_{I_{H}^{2}}^{2} \\
& +\left(m c+K_{F_{1}, H}^{1 / 2}\right)^{2}\left(m+K_{\lambda} \alpha^{-1}\right)\left(2 \alpha \delta C_{\delta}\right)^{-1}\left|\varphi_{2}\right|_{I_{H}^{2}}^{2} \\
& +\left(m+\frac{3 K_{F_{1}, H}}{\lambda}+K_{G_{0}, H}+\frac{3 K_{G_{1}}}{\lambda} K_{G_{0}, H}\right) \int_{0}^{t} \mathrm{e}^{m s} E|v(s)|^{2} \mathrm{~d} s \\
& +\left(m+K_{\lambda} \alpha^{-1}\right)\left(2 \alpha \delta C_{\delta}\right)^{-1}\left(2 \alpha \delta+\left(m c+K_{F_{1}, H}^{1 / 2}\right)^{2}\right) \int_{0}^{t} \mathrm{e}^{m s} E|v(s)|^{2} \mathrm{~d} s \\
& +c_{B}^{2}\left(2 \alpha \delta C_{\delta}\right)^{-1}\left(m+K_{\lambda} \alpha^{-1}\right) \int_{0}^{t} \mathrm{e}^{m s} E\|v(s)\|^{2} \mathrm{~d} s .
\end{aligned}
$$

Thanks now to conditions in this theorem, for $m>0$ small enough, we deduce

$$
\begin{aligned}
0< & 1-c\left(m+K_{\lambda} \alpha^{-1}\right)\left(2 \alpha^{1 / 2} C_{\delta}\right)^{-1} \\
\frac{\lambda}{3}> & c^{2}\left(m+\frac{3 K_{F_{1}, H}}{\lambda}+K_{G_{0}, H}+\frac{3 K_{G_{1}}}{\lambda} K_{G_{0}, H}\right) \\
& +\left(m+K_{\lambda} \alpha^{-1}\right)\left(2 \alpha \delta C_{\delta}\right)^{-1}\left(2 \alpha \delta c^{2}+\left(m c^{2}+c K_{F_{1}, H}^{1 / 2}\right)^{2}+c_{B}^{2}\right),
\end{aligned}
$$

hence we have ensured that the null solution to problem $(Q)$ is exponentially stable in mean square.

Remark 7 It is also possible to prove that, if the solution $(u, v)$ to $(Q)$ is exponentially stable in mean square then, the null solution to problem $(Q)$ is almost surely exponentially 
stable. To this end, we can first establish a bound for $E\left[\sup _{N \leq t \leq N+1}\left(|v(t)|^{2}+\|u(t)\|^{2}\right)\right]$, for any $N \in \mathbb{N}$, similar to that one in (12). To this end, we point out that one must use hypothesis $(H)$ in the particular case $m=0$.

\section{Examples}

To illustrate our theory we are going to exhibit a couple of examples. The first corresponds to the situation in which $B(t, \cdot): H \rightarrow H$, and, as a consequence, prove that our theory improves a classical result on the asymptotic behaviour of partial differential equations of second order in time without hereditary characteristics obtained due to Curtain [6].

\section{Example 8}

Consider the lateral displacement of a stretched string subjected to a random loading with delays:

$$
\left\{\begin{array}{l}
\frac{\partial^{2} u}{\partial t^{2}}-\frac{\partial^{2} u}{\partial x^{2}}+\vartheta \frac{\partial u}{\partial t}=\sigma \frac{\partial u(t-\tau(t))}{\partial x} \frac{d W(t)}{d t}, \text { in }(0,+\infty) \times(0,1), \\
u(t, 0)=u(t, 1)=0, t \in(0,+\infty), \\
u(0, x)=u_{0}, \quad \frac{\partial u}{\partial t}(0, x)=v_{0}(x), \text { in }(0,1), \\
u(t)=\varphi_{1}(t), \quad \frac{\partial u(t)}{\partial t}=\varphi_{2}(t), t \in(-h, 0),
\end{array}\right.
$$

where $\vartheta>0, \sigma \in \mathbb{R}, W(t)$ is a one-dimensional Wiener process, and $\tau \in C^{1}\left(\mathbb{R}^{+}\right)$is such that $0 \leq \tau(t) \leq h, \forall t \geq 0$, being $\tau^{*}=\sup _{t \geq 0} \tau^{\prime}(t)<1$.

This example can be set within our formulation by taking $H=L^{2}(0,1), V=H_{0}^{1}(0,1)$,

$$
A(t) u(t)=-\frac{\partial^{2} u}{\partial x^{2}}(t), B(t, v(t))=\vartheta v(t) \text { and } G_{0}(t, \xi, \eta)=\sigma \frac{\partial \xi(-\tau(t))}{\partial x} .
$$

If we denote $\theta(t)=t-\tau(t)$, then there exists $k>0$ such that $\theta^{-1}(t) \leq t+k, \forall t \geq-\tau(0)$, and consequently, with the notation above, it is easy to check all the conditions and deduce that

$$
\beta=c_{B}=\vartheta, \alpha=1, c=\frac{1}{\pi}, K_{G_{0}, H}\left(m_{0}\right)=0, K_{G_{0}, V}\left(m_{0}\right)=\frac{\sigma^{2}}{1-\tau^{*}} e^{m_{0} k},
$$

with $m_{0}>0$ arbitrarily chosen.

Thus, we can ensure that given $\varphi_{1} \in I^{2}(-h, 0 ; V), \varphi_{2} \in I^{2}(-h, 0 ; H), u_{0} \in L^{2}\left(\Omega, \mathrm{F}_{0}, P ; V\right)$ and $v_{0} \in L^{2}\left(\Omega, \mathcal{F}_{0}, P ; H\right)$, there exists a unique solution $u \in I^{2}(-h, T ; V) \cap L^{2}(\Omega ; C(0, T ; V))$, 
$\frac{\partial u}{\partial t} \in I^{2}(-h, T ; H) \cap L^{2}(\Omega ; C(0, T ; H))$ to the corresponding problem $(P)$, and using (14) we immediately obtain the exponential stability in mean square and pathwise of the solution to our problem provided

$$
\frac{\sigma^{2}}{1-\tau^{*}}<\min \left\{\pi, \frac{2 \vartheta \pi^{2}}{2 \pi^{2}+\vartheta^{2}}\right\}=\frac{2 \vartheta \pi^{2}}{2 \pi^{2}+\vartheta^{2}} .
$$

Observe that in the particular case in which $\tau(t)=h$ for all $t \geq 0$, the previous condition reads $\sigma^{2}<\frac{2 \vartheta \pi^{2}}{2 \pi^{2}+\vartheta^{2}}$, a better estimate than the condition $\sigma^{2}<\frac{4 \vartheta \pi^{2}}{4 \pi^{2}+\vartheta\left(\vartheta+\sqrt{\vartheta^{2}+4 \pi^{2}}\right)}$, obtained by Curtain in [6] in the case without delays, i.e., when $h=0$.

\section{Example 9}

Consider the problem

$$
\left\{\begin{array}{l}
\frac{\partial^{2} u}{\partial t^{2}}-\frac{\partial^{2} u}{\partial x^{2}}-\gamma \frac{\partial^{2}}{\partial x^{2}}\left(\frac{\partial u}{\partial t}\right)-\frac{\partial}{\partial x}\left(k\left(t, \frac{\partial^{2} u}{\partial x \partial t}\right)\right) \\
=f_{1}\left(t, u\left(t-\rho_{1}(t)\right), \frac{\partial u}{\partial x}\left(t-\rho_{2}(t)\right), \frac{\partial u}{\partial t}\left(t-\rho_{3}(t)\right)\right) \\
+\left(g_{1}\left(t, u\left(t-\rho_{4}(t)\right), \frac{\partial u}{\partial x}\left(t-\rho_{5}(t)\right), \frac{\partial u}{\partial t}\left(t-\rho_{6}(t)\right), \frac{\partial}{\partial x}\left(\frac{\partial u}{\partial t}\left(t-\rho_{7}(t)\right)\right)\right)\right. \\
\left.+g_{0}\left(t, \frac{\partial u\left(t-\rho_{8}(t)\right)}{\partial x}, \frac{\partial u\left(t-\rho_{9}(t)\right)}{\partial t}\right)\right) \frac{d W(t)}{d t}, \text { in }(0,+\infty) \times(0, \pi) \\
u(t, 0)=u(t, \pi)=0, t \in(0,+\infty), \\
u(0, x)=u_{0}, \quad \frac{\partial u}{\partial t}(0, x)=v_{0}(x), \text { in }(0, \pi) \\
u(t)=\varphi_{1}(t), \quad \frac{\partial u(t)}{\partial t}=\varphi_{2}(t), t \in(-h, 0)
\end{array}\right.
$$

where $\gamma, h>0$, and $W(t)$ is a one-dimensional Wiener process. Let us set $H=L^{2}(0, \pi)$, $V=H_{0}^{1}(0, \pi)$, and consider $A(t) u(t)=-\frac{\partial^{2} u}{\partial x^{2}}(t), \forall u \in V, \forall t \geq 0$. On the other hand, we assume that $k: \mathbb{R}^{+} \times \mathbb{R} \rightarrow \mathbb{R}$ is a continuous map such that there exists $c_{k}>0$ such that

$$
(k(t, x)-k(t, \widetilde{x}))(x-\widetilde{x}) \geq 0,|k(t, x)| \leq c_{k}|x| \quad \forall x, \widetilde{x} \in \mathbb{R}, \forall t \geq 0 .
$$

Then we consider

$$
\langle B(t, v), w\rangle=\gamma \int_{0}^{\pi} \frac{\mathrm{d} v}{\mathrm{~d} x} \frac{\mathrm{d} w}{\mathrm{~d} x} d x+\int_{0}^{\pi} k\left(t, \frac{\mathrm{d} v}{\mathrm{~d} x}\right) \frac{\mathrm{d} w}{\mathrm{~d} x} d x, \forall v, w \in V, \forall t \geq 0 .
$$

Assume that $\rho_{i} \in C^{1}\left(\mathbb{R}^{+}\right), i=1, \cdots, 9$ are measurable functions such that $0 \leq \rho_{i}(t) \leq h$, $\forall t \in \mathbb{R}^{+}$, being $\rho^{*}=\max _{1 \leq i \leq 9}\left(\max _{t \in \mathbb{R}^{+}} \rho_{i}^{\prime}(t)\right)<1$. As in the previous example, if we denote $\theta_{\rho_{i}}(t)=t-\rho_{i}(t)$, then there exists $k_{\rho}>0$ such that $\theta_{\rho_{i}}^{-1}(t) \leq t+k_{\rho}, \forall t \geq-\min _{1 \leq i \leq 9} \rho_{i}(0)$. 
We suppose that $f_{1}: \mathbb{R}^{+} \times \mathbb{R}^{3} \rightarrow \mathbb{R}$ is a measurable function such that $f_{1}(t, 0,0,0)=0$, and there exists $L_{f_{1}}>0$ such that

$$
\left|f_{1}(t, a, y, b)-f_{1}(t, \widetilde{a}, \widetilde{y}, \widetilde{b})\right| \leq L_{f_{1}}(|a-\widetilde{a}|+|y-\widetilde{y}|+|b-\widetilde{b}|),
$$

$\forall a, \widetilde{a}, b, \widetilde{b}, y, \widetilde{y} \in \mathbb{R}, \forall t \in \mathbb{R}^{+}$. We denote by $F_{1}(t, \cdot, \cdot)$ the family defined by

$$
\left\langle F_{1}(t, \xi, \eta), v\right\rangle=-\int_{0}^{\pi} f_{1}\left(t, \xi\left(-\rho_{1}(t)\right)(x), \frac{\partial \xi}{\partial x}\left(-\rho_{2}(t)\right)(x), \eta\left(-\rho_{3}(t)\right)(x)\right) \frac{d v}{d x}(x) d x,
$$

$\forall(\xi, \eta) \in C(-h, 0 ; V) \times C(-h, 0 ; H), \forall v \in V$, for each $t \in \mathbb{R}^{+}$.

Let $g_{0}: \mathbb{R}^{+} \times \mathbb{R}^{2} \rightarrow \mathbb{R}$ be a Lipschitz continuous function such that $g_{0}(t, 0,0)=0$, with

$$
\left|g_{0}(t, x, y)-g_{0}(t, \widetilde{x}, \widetilde{y})\right|^{2} \leq L_{g_{0}}^{2}\left(|x-\widetilde{x}|^{2}+|y-\widetilde{y}|^{2}\right), \quad \forall(x, y),(\widetilde{x}, \widetilde{y}) \in \mathbb{R}^{2}, \forall t \in \mathbb{R}^{+} .
$$

We consider

$$
G_{0}(t, \xi, \eta)=g_{0}\left(t, \frac{\partial \xi\left(-\rho_{8}(t)\right)}{\partial x}, \eta\left(-\rho_{9}(t)\right)\right), \quad \forall(t, \xi, \eta) \in \mathbb{R}^{+} \times C(-h, 0 ; V) \times C(-h, 0 ; H) .
$$

Suppose also that $g_{1}: \mathbb{R}^{+} \times \mathbb{R}^{4} \rightarrow \mathbb{R}$ is a measurable function such that $g_{1}(t, 0,0,0,0)=0$, and there exists $L_{g_{1}}>0$ such that

$$
\left|g_{1}(t, a, y, b, z)-g_{1}(t, \widetilde{a}, \widetilde{y}, \widetilde{b}, \widetilde{z})\right|^{2} \leq L_{g_{1}}\left(|a-\widetilde{a}|^{2}+|y-\widetilde{y}|^{2}+|b-\widetilde{b}|^{2}+|z-\widetilde{z}|^{2}\right),
$$

$\forall a, \widetilde{a}, b, \widetilde{b}, y, \widetilde{y}, z, \widetilde{z} \in \mathbb{R}, \forall t \in \mathbb{R}^{+}$. For every $t \in \mathbb{R}^{+}$and $\xi, \eta \in C(-h, 0 ; V)$, let $G_{1}(t, \xi, \eta)$ be the element of $H$ given, a.e. $x \in \mathcal{O}$, by

$$
G_{1}(t, \xi, \eta)(x)=g_{1}\left(t, \xi\left(-\rho_{4}(t)\right)(x), \frac{\partial \xi}{\partial x}\left(-\rho_{5}(t)\right)(x), \eta\left(-\rho_{6}(t)\right)(x), \frac{\partial \eta}{\partial x}\left(-\rho_{7}(t)\right)(x)\right),
$$

Then, it is easy to check that if, for example

$$
\frac{L_{g_{1}}}{1-\sigma^{*}} \leq \gamma e^{-\gamma T}
$$

we can assure existence and uniqueness of a solution to the corresponding problem (see Garrido-Atienza [7]). In particular, for $\varphi_{1} \in I^{2}(-h, 0 ; V), \varphi_{2} \in I^{2}(-h, 0 ; H), u_{0} \in L^{2}\left(\Omega, \mathcal{F}_{0}, P ; V\right)$ and $v_{0} \in L^{2}\left(\Omega, \mathcal{F}_{0}, P ; H\right)$ given, there exists a unique solution $u \in I^{2}(-h, T ; V) \cap L^{2}(\Omega ; C(0, T ; V))$, $\frac{\partial u}{\partial t} \in I^{2}(-h, T ; H) \cap L^{2}(\Omega ; C(0, T ; H))$. The constants are now

$$
\begin{gathered}
c_{B}=c_{k}, \beta=\gamma, \alpha=c=1, K_{F_{1}, H}\left(m_{0}\right)=3 e^{-m_{0} k \rho} \frac{L_{f_{1}}}{1-\rho^{*}}, K_{F_{1}, V}\left(m_{0}\right)=6 \pi e^{-m_{0} k \rho} \frac{L_{f_{1}}}{1-\rho^{*}}, \\
K_{G_{0}, V}\left(m_{0}\right)=K_{G_{0}, H}\left(m_{0}\right)=2 e^{-m_{0} k_{\rho}} \frac{L_{g_{0}}^{2}}{1-\rho^{*}}, K_{G_{1}}=e^{-m_{0} k_{\rho}} \frac{L_{g_{1}}^{2}}{1-\rho^{*}}
\end{gathered}
$$

The solution is exponentially stable in mean square and almost surely if we impose the corresponding hypothesis (15). 


\section{Concluding remarks}

Some results on the exponential stability of functional stochastic partial differential equations of second order in time have been proved, these extend some results from [3]. Moreover, in the particular case without delay, a stability criterium in [6] is also improved (see Example 8).

Another point is that, although we have only considered the case of a real Wiener process, the results can be extended to a Hilbert valued situation. However, we have preferred to consider this framework for the sake of clarity.

Acknowledgment.

This work has been partially supported by Junta de Andalucía Project FQM314, and by Ministerio de Ciencia y Tecnología (Spain), projects HA2001-0075 and BFM2002-03068.

\section{References}

[1] T. Caraballo, Asymptotic exponential stability of stochastic partial differential equations with delay, Stochastics Stochastics Rep. 33 (1990), 27-47.

[2] T. Caraballo, M.J. Garrido-Atienza and J. Real, Asymptotic stability of non-linear stochastic evolution equations, Stoch. Anal. Appl. 21 (2003), no. 2, 301-327.

[3] T. Caraballo, M.J. Garrido-Atienza and J. Real, The exponential behaviour of nonlinear stochastic functional equations of second order in time, Stochastics and Dynamics 3 (2003), no. 2, 169-186.

[4] T. Caraballo and K. Liu, On exponential stability criteria of stochastic partial differential equations, Stochastic Processes and their Applications 83 (1999), 289-301.

[5] T. Caraballo, K. Liu and A. Truman, Stochastic functional partial differential equations: existence, uniqueness and asymptotic decay property, Proc. R. Soc. Lond. A 456 (2000), 1775-1802.

[6] R.F. Curtain, Stability of stochastic partial differential equation, J. Math. Anal. Appl. 79 (1981), 352-369.

[7] M.J. Garrido-Atienza, "Algunos resultados de existencia, unicidad y estabilidad para EDP funcionales estocásticas no lineales", PhD. Thesis, Universidad de Sevilla, 2002. 
[8] M.J. Garrido-Atienza, J. Real, Existence and uniqueness of solutions for delay stochastic evolution equations of second order in time, Stochastics and Dynamics 3 (2003), no. 2, 141-167.

[9] H. Lisei, Conjugation of flows for stochastic and random functional differential equations, Stochastics and Dynamics 1 (2001), no. 2, 283-298.

[10] K. Liu, Lyapunov functionals and asymptotic stability of stochastic delay evolution equations, Stochastics Stochastics Rep. 63 (1998) 1-26.

[11] K. Liu and X.R. Mao, Exponential stability of nonlinear stochastic evolution equations, Stochastic Processes and their Applications 78 (1998), 173-193.

[12] T. Taniguchi, Asymptotic stability theorems of semilinear stochastic evolution equations in Hilbert spaces, Stochastics Stochastics Rep. 53 (1995), no. 1-2, 41-52.

[13] T. Taniguchi, K. Liu and A. Truman, Existence, uniqueness, and asymptotic behavior of mild solutions to stochastic functional differential equations in Hilbert spaces, $J$. Differential Equations 181 (2002), no. 1, 72-91. 